\title{
Tumor associated PD-L1 expression pattern in microscopically tumor positive sentinel lymph nodes in patients with melanoma
}

\author{
Ahmad A. Tarhini ${ }^{1}{ }^{2 *}$, Haris Zahoor ${ }^{2}$, Jennifer H. Yearley ${ }^{3}$, Christopher Gibson ${ }^{3}$, Zahra Rahman², Rachel Dubner ${ }^{2}$, \\ Uma N. M. Rao ${ }^{1,2}$, Cindy Sander ${ }^{1}$ and John M. Kirkwood ${ }^{1,2}$
}

\begin{abstract}
Background: Characterization of PD-L1 expression within clinically/radiologically negative but microscopically tumor positive sentinel lymph nodes (SLN) is important to our understanding of the relevance of this immune checkpoint pathway for adjuvant therapy.

Methods: Patients included had primary cutaneous melanoma, Breslow thickness of 2.01-4.0 or $>4 \mathrm{~mm}$ with or without tumor ulceration (T3a, T3b, T4a, T4b). All patients had microscopically tumor positive SLN. Hematoxylin and eosin (H\&E) staining was performed, followed by PD-L1 immunohistochemical (IHC) staining using a preliminary IHC assay with anti-PD-L1 antibody clone 22C3. The slides were separately evaluated by two pathologists (JY and CG). Samples containing metastatic melanoma lesions were scored separately for PD-L1 expression in intratumoral and peritumoral locations, by utilizing two scoring methods.

Results: Twenty-four patients where metastatic melanoma presence in the SLN was confirmed by H\&E review of the cut sections were included in the final analysis of PD-L1 expression. SLN tumor size ranged from 1 to $2 \mathrm{~mm}$. For three patients, the melanin content was too high to confidently assign a PD-L1 score. For the remaining 21 patients, all had some evidence of either intratumoral or peritumoral PD-L1 expression. The frequency of intratumoral tumorassociated PD-L1 expression was: $0 \%$ of tumor cells (3 pts, $14 \%) ;<1 \%(5$ pts, $24 \%) ; 1-10 \%$ (6 pts, $29 \%$ ) and >10 \% (7 pts, $33 \%)$.

Conclusions: Tumor-associated PD-L1 expression is readily detectable within melanoma micrometastases in the SLN of the majority of patients. These results support the testing of a therapeutic role for PD1/PD-L1 inhibition in the adjuvant setting, targeting melanoma micrometastases.
\end{abstract}

Keywords: Melanoma, PD-1, PD-L1, Sentinel lymph node

\section{Background}

PD-1 is an immune-inhibitory receptor belonging to CD28/CTLA4 receptor family that is expressed on activated $T$ cells, $B$ cells and monocytes [1,2]. PD-1 is also expressed on $\mathrm{T}$ regulatory cells where it interacts with

\footnotetext{
*Correspondence: tarhiniaa@upmc.edu

${ }^{2}$ University of Pittsburgh Cancer Institute, 5150 Centre Avenue,

Pittsburgh, PA 15232, USA

Full list of author information is available at the end of the article
}

dendritic cells and NK T cells, and shown to be associated with anergy and tumor immune escape [3-5]. The role of PD-1 as a negative regulator of $\mathrm{T}$ cell activity is mediated through its interaction with its ligands PD-L1 and PD-L2 (also known as B7-H1 and B7-H2 based on the similarity to other B7 family molecules) that are expressed on immune cells and tumor cells [6]. PD-L1 is expressed on $\mathrm{T}$ and $\mathrm{B}$ cells, macrophages and dendritic cells [7]. PD-L1 and PD-L2 are expressed on many human tumors including melanoma, glioblastoma, non-small cell lung cancer 
and urothelial, ovarian, breast, cervical, colon, pancreatic and gastric carcinoma [8-18]. PD-L1 has been implicated in tumor immune escape from the host immune system and in mediating tumor anti-apoptotic activity [2, 19-21]. PD-1 ligand 1 and 2 (PD-Ls) expressed on antigen-presenting cells have been shown to indirectly induce $\mathrm{T}$ cell anergy or exhaustion via PD-1 on T cells, whereas PD-L1 expressed on peripheral tissues directly suppresses self-reactive lymphocytes [22, 23]. PD-Ls expressed on tumors regulate the generation of adaptive regulatory $\mathrm{T}$ cells resulting in tumor-induced immune suppression [5], including the suppression of the effector function of CD8+ T cells [21]. Interestingly, a significant inverse correlation was observed between PD-L1 expression and the intraepithelial CD8+ T lymphocyte count, suggesting that PD-L1 on tumor cells directly suppresses antitumor CD8+ T cells [9]. PD-1 blockade has been shown to enhance the expansion and functional capacity of human melanoma antigen specific cytotoxic T cells [24]. Clinically, higher expression levels of PD-L1 on tumors have been shown to correlate with poor prognosis in several malignant tumors including melanoma, esophagus, kidney, lung, and brain, pancreatic, ovarian and head and neck $[8,9,12,16,25,26]$. These data illustrate a central role for the PD-1/PD-L1 axis in tumor immune escape and have led to the clinical targeting of PD-1 and PD-L1 as an antitumor strategy.

Pembrolizumab and nivolumab, both of which target PD-1, have been approved for the treatment of metastatic melanoma. Approval of pembrolizumab was granted based on data from a cohort of a phase I trial (KEYNOTE-002) in which 411 patients with advanced melanoma who were refractory to ipilimumab or were ipilimumab naive received pembrolizumab at 2 or $10 \mathrm{mg} /$ $\mathrm{kg}$ every 3 weeks or $10 \mathrm{mg} / \mathrm{kg}$ every 2 weeks [27, 28]. Overall response rates were 40 and $28 \%$ in ipilimumabnaive and ipilimumab-refractory treatment arms, respectively. In addition, response rates and progression-free survival were significantly higher in patients with high PD-L1 tumor expression compared with patients who were considered PD-L1 negative [29]. In December 2014, nivolumab became the second monoclonal antibody targeting the PD-1 receptor to be approved by FDA for the treatment of patients with unresectable or metastatic melanoma and disease progression following ipilimumab and a BRAF inhibitor [30]. Approval for nivolumab was based on results from the first 120 patients enrolled in a phase III trial (CheckMate 037) testing nivolumab versus either dacarbazine or carboplatin/paclitaxel in patients with metastatic melanoma who progressed on or after anti-CTLA-4 therapy and a BRAF inhibitor (if BRAF V600 mutation positive). The overall tumor response rates were 32 and $11 \%$ in favor of nivolumab [31]. The use of nivolumab in previously untreated metastatic patients has also shown excellent activity; objective response rate of $40.0 \%$ as compared to $13.9 \%$ in the dacarbazine group [32].

The significant clinical activity of anti-PD1 antibodies has supported their planned testing as adjuvant therapy in patients with operable melanoma at high risk for relapse and death from melanoma. Adjuvant therapy targets micrometastatic disease which is the source of future mortality from melanoma recurrence and presents an opportunity for curing this disease. We hypothesized that micrometastatic tumors that are the source of future melanoma relapse in high risk patients express PD-L1 making them susceptible to PD1/PD-L1 therapeutic blockade. Characterization of PD-L1 expression within clinically/radiologically negative but microscopically tumor positive sentinel lymph nodes (SLN) is important to our understanding of the relevance of this immune checkpoint pathway for adjuvant therapy. In this report, we present data which shows that tumor-associated PD-L1 expression is readily detectable within melanoma micrometastases in the SLN.

\section{Methods}

\section{Patients}

Twenty-four patients with primary cutaneous melanoma were included in this study. All patients had a primary tumor Breslow thickness of 2.01-4.00 mm without (T3a) or with ulceration (T3b), or $>4 \mathrm{~mm}$ without (T4a) or with ulceration (T4b). Patients had known microscopically tumor positive SLN detected during standard SLN biopsy procedures. All patients provided a written informed consent. Table 1 summarizes patient demographics and baseline disease characteristics.

\section{Procedures}

Cut sections $(5 \mu \mathrm{m})$ were obtained from formalin-fixed, paraffin-embedded (FFPE) SLN tissue from patients enrolled on this study. Slides were first stained with haematoxylin and eosin. PD-L1 immunostaining was performed using a preliminary immunohistochemistry (IHC) assay with anti-PD-L1 antibody clone 22C3. Slides from two patients were also stained using an antiHMB45/MelA protocol to better ascertain the presence and/or localization of melanoma lesions in the tissue in order to facilitate interpretation of the PD-L1 staining in those samples. All staining was performed on Dako autostainers at Merck Research Laboratories, Palo Alto, CA. The anti-PD-L1 antibody clone $22 \mathrm{C} 3$ is a mouse antihuman PD-L1 IgG1k generated through murine immunization with a fusion protein containing the human extra 
Table 1 Patient demographics and baseline disease characteristics $(\mathbf{N}=\mathbf{2 4}$ patients)

\begin{tabular}{lc}
\hline Variable & No. of patients (\%) \\
\hline Age, years; median (range) & $58(18-75)$ \\
Cutaneous primary & $24(100)$ \\
Gender & \\
Female & $12(50)$ \\
Male & $12(50)$ \\
Performance status (ECOG) & \\
0 & $18(75)$ \\
1 & $6(25)$ \\
AJCC stage & \\
IIIA & $5(21)$ \\
IIIB & $16(67)$ \\
IIIC & $3(12)$ \\
Ulceration of primary & \\
Yes & $18(75)$ \\
No & $6(25)$ \\
\hline
\end{tabular}

ECOG Eastern Cooperative Oncology Group, AJCC American Joint Committee on Cancer

cellular domain of PD-L1 and subsequent hybridoma formation [33].

The slides were separately evaluated by two pathologists. Samples containing metastatic melanoma lesions were scored separately for PD-L1 expression in intratumoral (including along tumor periphery but with clear tumor cell labeling) and peritumoral (expression external to tumor nodule in immediately surrounding tissue) locations. PD-L1 positivity was defined as partial or complete membrane staining of a tumor cell using the 22C3 antibody [33]. Two scoring methods were utilized: (1) semi-quantitative scoring method-samples containing metastatic melanoma lesions were scored separately for PD-L1 expression in intratumoral (including along tumor periphery but with clear tumor cell labeling) and peritumoral (expression external to tumor nodule in immediately surrounding tissue; immune cells) locations. For intratumoral signals, attempts were made to classify the expression as tumor cell associated (indicated by the letter " $T$ "), non-tumor cell associated (indicated by the letters "NT"), or both (indicated by "T/NT"). Scores were assigned using a $0-5$ semiquantitative scale assessing prevalence of positive cells where 0 , negative; 1 , minimal or rare; 2 , low; 3 , moderate; 4 , high; and 5 , very high. Samples where melanin content was too high to confidently assign a PD-L1 score were specifically noted. (2) Percentage estimates: scores at the low end were given a score of $0 \%$ of tumor cells, $<1,1$, and $3 \%$ (roughly indicates $>1 \%$ but $<5 \%$ ). Scores at the high end were given in $10 \%$ increments.

\section{Statistical analysis}

Descriptive statistics were used to tabulate and present the study findings.

\section{Results}

Twenty-four patients where metastatic melanoma presence in the SLN was confirmed by H\&E review of the cut sections were included in the final analysis of PD-L1 expression. Table 1 summarizes patient demographics and disease characteristics. Table 2 summarizes the results of tumor associated PD-L1 expression. Three patient samples were noted to have high melanin content where it was difficult to assign a PD-L1 expression score.

Of the remaining 21 patient samples, 13 (62\%) showed PD-L1 positivity defined as partial or complete membrane staining in $\geq 1 \%$ (range 1-90\%) of tumor cells using the 22C 3 antibody. The majority of patient samples (20 samples; $95 \%$ ) showed some degree of intratumoral or peritumoral PD-L1 expression. Table 3 presents the frequency of tumor-associated PD-L1 expression by the percentage of expression. Among patient samples that were considered PD-L1 positive, more than half were scored at $>10 \%$. Figure 1 shows examples of SLN melanoma sample intratumoral PD-L1 immunohistochemical staining, both positive (A) and negative (B). Finally, we attempted to score the non-tumor (lymphoid tissue) associated PD-L1 expression in the 24 patient SLN samples included in this study. PD-L1 expression in these areas was classified as (1) expression by histiocytes in the sinuses of the node and (2) expression observed in nonsinus lymphoid tissue with morphologic characteristics consistent with antigen presenting cell populations (dendritic cells and macrophages). Table 4 summarizes these observations.

\section{Results and discussion}

In this study, we have reported that melanoma patients with SLN micrometastases detected by SLN biopsy demonstrate evidence of tumor associated PD-L1 expression. The majority of patients were found to have tumor associated PD-L1 expression estimated by two scoring methods, including the percentage estimates method utilized in prior studies in patients with metastatic melanoma [34]. Inhibition of the PD-1/PD-L1 axis has emerged as an important immune checkpoint therapeutic strategy with unprecedented clinical responses seen in patients with metastatic melanoma. Efforts are underway to test anti-PD1 monoclonal antibodies as adjuvant therapy in patients with operable melanoma who continue to carry a high risk of recurrence and death after surgery. In these patients, residual micrometastatic disease is expected to be the source of future melanoma relapse. For patients with AJCC stages IIB-C/III/IV, melanoma carries a high 
Table 2 Tumor associated PD-L1 expression

\begin{tabular}{|c|c|c|c|}
\hline \multirow[t]{3}{*}{ Patient with SLN+ $(\mathrm{N}=24)$} & \multicolumn{3}{|l|}{ Tumor associated expression } \\
\hline & \multicolumn{2}{|l|}{ Semi-quantitative scoring } & \multirow[t]{2}{*}{ Percentage estimates-tumor } \\
\hline & PD-L1 score-tumor & PD-L1_peritumoral & \\
\hline 1 & $1 \mathrm{~T}$, very weak & 1 & 3 \\
\hline 2 & 4, predominantly $T$, peripheral & 1 & 40 \\
\hline 3 & Melanin confounds & 2 & NA \\
\hline 4 & 0 & 2 (adjacent subcapsular sinus) & 0 \\
\hline 5 & $4 \mathrm{~T} / \mathrm{NT}$ & 2 & 30 \\
\hline 6 & 2, predominantly NT & 3 & 3 \\
\hline 7 & 0 & 3 (adjacent subcapsular sinus) & 0 \\
\hline 8 & 2.5 , predominantly NT & 3 & 10 \\
\hline 9 & 4.5, predominantly $T$, weak & 2 (adjacent sinus) & 50 \\
\hline 10 & Melanin confounds & 0 & NA \\
\hline 11 & $1 \mathrm{~T}$ & 2.5 & $<1$ \\
\hline 12 & $0^{\mathrm{a}}$ & 2 & 0 \\
\hline 13 & $1 \mathrm{~T}$ & 1 & $<1$ \\
\hline 14 & $2.5 \mathrm{NT}$ & 2 & 10 \\
\hline 15 & 2 predominantly NT & 3 & 3 \\
\hline 16 & $1 \mathrm{~T}$ & 2 & 1 \\
\hline 17 & Melanin confounds & 0 & NA \\
\hline 18 & 1 & 1 & $<1$ \\
\hline 19 & 1 NT & 2.5 & $<1$ \\
\hline 20 & $2 T ?$, melanin confounds & 2 & $<1$ \\
\hline 21 & 4, predominantly $T$, peripheral & 3.5 & 40 \\
\hline 22 & 5 , predominantly $\top$ & 2 & 90 \\
\hline 23 & 4 & 0 & 40 \\
\hline 24 & $4.5 \mathrm{~T} / \mathrm{NT}$ & 0 & 70 \\
\hline
\end{tabular}

a Very few tumor cells. In one region where a few tumor cells were present, a dense area of PD-L1 positivity was present directly adjacent but was impossible to say if all were non-tumor; this is interpreted as most likely

Table 3 The frequency of tumor-associated PD-L1 expression by percentage of expression (excluding samples where melanin content was too high to confidently assign a PD-L1 score; $\mathbf{N}=\mathbf{2 1}$ )

\begin{tabular}{lll}
\hline $\begin{array}{l}\text { Percentage esti- } \\
\text { mates }\end{array}$ & $\begin{array}{l}\text { No. of patients } \\
(\mathbf{N}=\mathbf{2 1})\end{array}$ & $\begin{array}{l}\text { Percentage of patient } \\
\mathbf{( N = 2 1 )}\end{array}$ \\
\hline 0 & 3 & 14 \\
$<1$ & 5 & 24 \\
$1-10$ & 6 & 29 \\
$>10$ & 7 & 33 \\
\hline
\end{tabular}

risk for recurrence and death from melanoma with surgical management alone [35-38]. Systemic adjuvant therapy that targets melanoma micrometastases is indicated postoperatively where it may provide the greatest opportunity for cure before relapse into advanced inoperable stages. Multiple systemic therapeutic agents have been tested as adjuvant therapy for melanoma with durable benefits seen only with high dose interferon-alfa (HDI) to date [39]. CTLA4-blockade with ipilimumab is currently being tested in the adjuvant EORTC 18071 trial (stage III; compared to placebo) and U.S. Intergroup E1609 (stage III and IV; compared to HDI). Ongoing adjuvant trials are also targeting patients with BRAF mutant melanoma including vemurafenib (BRIM-8) and dabrafenib/ trametinib (COMBI-AD). Adjuvant trials involving PD1blockade are expected to be activated in the second quarter of 2015. In this study, we have characterized PD-L1 expression within clinically/radiologically negative but microscopically tumor positive SLN, which may be important to our understanding of the relevance of this immune checkpoint pathway for adjuvant therapy.

The findings of this study further support the investigation of PD-1/PD-L1 blockade in the adjuvant setting in melanoma patients with high risk for relapse.

Previous studies have shown that increased tumor associated expression of PD-L1 is associated with a higher likelihood of clinical response to PD-1/PD-L1 blockade 


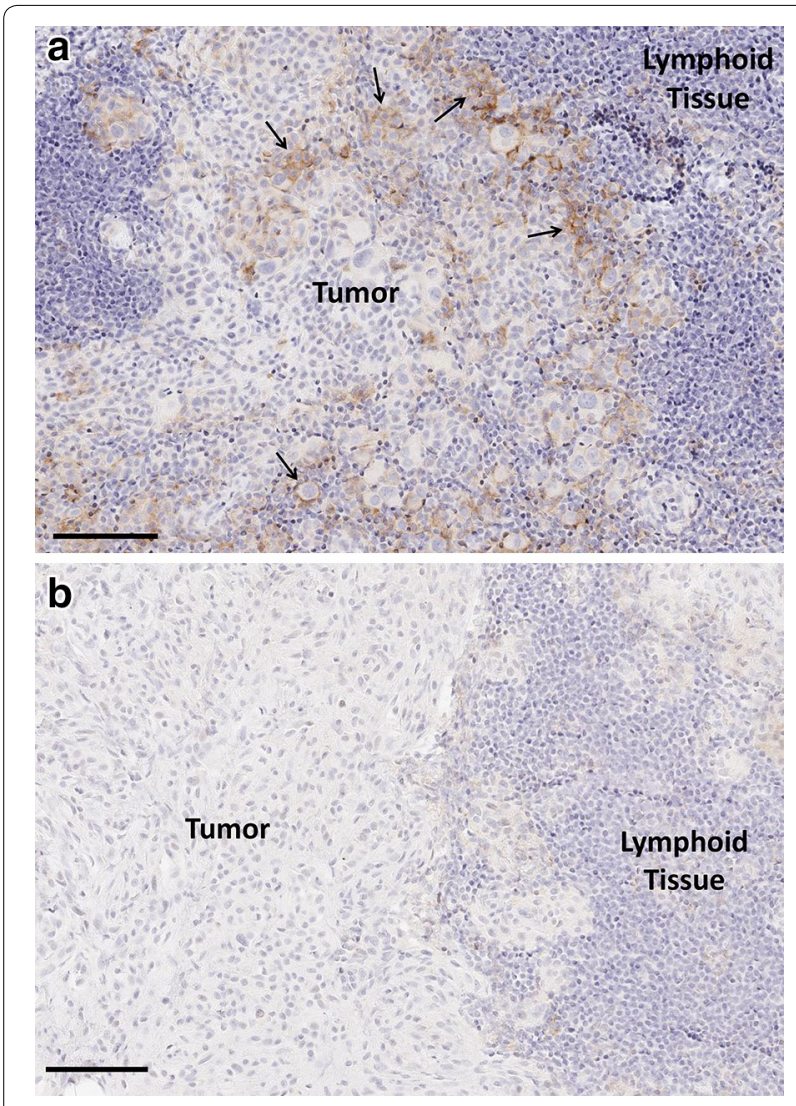

Fig. 1 Examples of sentinel lymph node melanoma sample intratumoral PD-L1 immunohistochemical staining, including positive for PD-L1 expression (brown chromogen) (a) and negative (b). The arrows point to representative areas of membranous staining interpreted as tumor cell expression. The scale bar represents $100 \mu \mathrm{m}$

[40-42]. Kefford et al., reported that melanoma patients receiving pembrolizumab in the phase I KEYNOTE-001 trial had differential clinical responses to treatment based on the baseline tumor PD-L1 expression pattern [34]. PD-L1 positivity in pre-treatment biopsies was defined as partial or complete membrane staining in $\geq 1 \%$ of tumor cells using the 22C3 antibody [34]. Patients with PD-L1 positive tumors had a $49 \%$ overall response rate (ORR) as compared to $13 \%$ in those with PD-L1 negative tumors. In the phase I study that tested multiple doses of nivolumab in advanced melanoma, an exploratory analysis to investigate tumor PD-L1 expression and its association with treatment response was also undertaken [43]. PD-L1 positivity was defined as tumor membrane staining with any intensity and with cut-off values of 1 and $5 \%$. ORR was superior in patients with a PD-L1 positive status. In the $1 \%$ cutoff analysis, $35 \%$ of patients considered PD-L1 positive had an objective response versus $13 \%$ in PD-L1 negative. The ORR increased (44\%) in PD-L1 positive patients by increasing the cutoff to $5 \%$ with no change in the ORR in the PD-L1 negative group. Overall survival (OS) in PD-L1 positive patients was 25 months as compared to 12 months in PD-L1 negative at $1 \%$ cutoff [43]. By increasing the cutoff to $5 \%$, median OS was not reached in the PD-L1 positive cohort as compared to 13 months in PD-L1 negative. Similarly, PFS was 9 months in PD-L1 positive patients as compared to 2 months in PD-L1 negative, at both 1 and $5 \%$ cutoff [43]. Similar findings have been reported in other tumor types in patients treated with anit-PD1 antibodies. For example, patients with non-small cell lung cancer (NSCLC) receiving nivolumab in a phase I clinical trial also showed correlation between clinical efficacy and tumor PD-L1 expression [44]. PD-L1 negative tumors showed no objective responses to nivolumab as compared to a $50 \%$ ORR in patients with PD-L1 positive status. PFS at 24 weeks was $70 \%$ and 1 -year OS of $80 \%$ in PD-L1 positive patients as compared to 57 and $71 \%$ in PD-L1 negative patients respectively [44]. The extent of PD-L1 expression was also found to correlate with ORR in a recent study of pembrolizumab in NSCLC with shorter progression-free and overall survival among patients with low tumor expression of PD-L1 [42]. Recently, PD-L1 expression in the immune infiltrate in the tumor has also been found to be associated with response to PD-1 pathway blockade therapy [45]. Interestingly, tumor PD-L1 negative status although it carries a lower likelihood of response to anti-PD1 antibodies, it does not preclude response and it is widely understood that the clinical utility of PD-L1 tumor expression requires refinement. However, these observations in patients with metastatic melanoma and other malignancies, support our hypothesis that micrometastatic tumors that are the source of future melanoma relapse in high risk patients express PD-L1 making them susceptible to PD1/PD-L1 therapeutic blockade.

Scoring of non-tumor associated PD-L1 expression in in the SLN was complicated by the fact that historical PD-L1 scoring has consistently centered on expression localized to and immediately surrounding tumor tissue. Normal or reactive lymphoid tissue is not typically included in scoring, so no experiential base exists for this type of analysis. In order to try to provide maximal information however, attempts were made to provide scores of PD-L1 expression in non-tumor infiltrated regions of lymph nodes where metastatic lesions were identified. PD-L1 expression in these areas could be classified as falling into two basic categories: (1) expression by histiocytes in the sinuses of the node and (2) expression observed in non-sinus lymphoid tissue with morphologic characteristics consistent with antigen presenting cell populations (dendritic cells and macrophages). Each of these categories was scored separately using a $0-5$ semiquantitative system, focused on signal prevalence. Given 
Table 4 Non-tumor associated lymphoid tissue PD-L1 expression

\begin{tabular}{|c|c|c|}
\hline \multirow[t]{2}{*}{ Patient with SLN+ } & \multicolumn{2}{|c|}{$\begin{array}{l}\text { Non-tumor associated lymphoid tissue } \\
\text { (semi-quantitative scoring method) }\end{array}$} \\
\hline & PD-L1-sinuses & PD-L1-APC pattern \\
\hline 1 & 4 & 3 \\
\hline 2 & 2 & 2 \\
\hline 3 & 4 & 2 \\
\hline 4 & 4 & 2.5 \\
\hline 5 & 2 & 2 \\
\hline 6 & 4 & 3 \\
\hline 7 & 5 & 4 \\
\hline 8 & 1 & 2 \\
\hline 9 & 3 & 3 \\
\hline 10 & 4 & 4 \\
\hline 11 & 1 & 4 \\
\hline 12 & 3 & 3 \\
\hline 13 & 2 & 2 \\
\hline 14 & 2 & 1 \\
\hline 15 & 3 & 3 \\
\hline 16 & 5 & 3 \\
\hline 17 & 4 (intense) & 3 \\
\hline 18 & 4 & 2.5 \\
\hline 19 & 3 & 2.5 \\
\hline 20 & 3 & 4 \\
\hline 21 & 3 & 4 \\
\hline 22 & 3 & 3 \\
\hline 23 & 4 & 4 (intense) \\
\hline 24 & 4 & 4 \\
\hline
\end{tabular}

the very different representation of tissue from sample to sample and the lack of historical experience with this type of non-tumor associated scoring by the evaluating pathologists, this data should be interpreted as extremely exploratory, and not suitable for drawing conclusions.

\section{Conclusions}

In conclusion, tumor-associated PD-L1 expression is readily detectable within melanoma micrometastases in the SLN of the majority of patients included in this study. These results support the testing of a therapeutic role for PD1/PD-L1 inhibition in the adjuvant therapy setting, targeting melanoma micrometastases. The testing of PD-L1 expression as a predictive biomarker for therapeutic benefit from anti-PD1 therapy is also warranted and to the best of our knowledge is planned in the context of ongoing adjuvant trials.

\section{Abbreviations}

AJCC: American Joint Committee on Cancer; CTLA-4: cytotoxic T-lymphocyte-associated protein 4; EORTC: European Organisation for Research and
Treatment of Cancer; FDA: Food and Drug Administration; HDI: high dose interferon-alfa; H\&E: hematoxylin and eosin; IHC: immunohistochemistry; ORR: overall response rate; OS: overall survival; PFS: progression free survival; PD1: programmed cell death protein 1; PD-L1: programmed death-ligand 1; SLN: sentinel lymph node; FFPE: formalin-fixed, paraffin-embedded.

\section{Authors' contributions}

AT and JY participated in study design, study conduct, data analysis, manuscript write up, data collection and final approval of manuscript. HZ, ZR, RD, UR and CS participated in study conduct, manuscript write up, data collection, and final approval of manuscript. CG participated in study conduct, data analysis, manuscript write up, data collection and final approval of manuscript. JK participated in manuscript write up, data collection and final approval of manuscript.

\section{Author details}

${ }^{1}$ University of Pittsburgh, Pittsburgh, PA, USA. ${ }^{2}$ University of Pittsburgh Cancer Institute, 5150 Centre Avenue, Pittsburgh, PA 15232, USA. ${ }^{3}$ Merck, Philadelphia, PA, USA.

\section{Acknowledgements}

This study was supported Merck and by National Institutes of Health award P50CA121973. University of Pittsburgh Cancer Institute shared resources that are supported in part by National Institutes of Health/National Cancer Institute award P30CA047904 were used for this project.

\section{Compliance with ethical guidelines}

\section{Competing interests}

Haris Zahoor, Zahra Rahman, Rachel Dubner, Uma N. M. Rao, Cindy Sander: Nothing to disclose. Jennifer A. Yearley, Christopher Gibson: Employed by Merck. Ahmad A. Tarhini and John M. Kirkwood: Receive research support from Merck, provide consulting services to Merck.

Received: 10 April 2015 Accepted: 21 September 2015

Published online: 30 September 2015

\section{References}

1. Freeman GJ, Long AJ, Iwai Y, Bourque $K$, Chernova T, Nishimura $H$, et al. Engagement of the PD-1 immunoinhibitory receptor by a novel B7 family member leads to negative regulation of lymphocyte activation. J Exp Med. 2000;192(7):1027-34

2. Iwai Y, Ishida M, Tanaka Y, Okazaki T, Honjo T, Minato N. Involvement of PD-L1 on tumor cells in the escape from host immune system and tumor immunotherapy by PD-L1 blockade. Proc Natl Acad Sci USA. 2002;99(19):12293-7. doi:10.1073/pnas.192461099.

3. Parekh W, Lalani S, Kim S, Halder R, Azuma M, Yagita H, et al. PD-1/ PD- $L$ blockade prevents anergy induction and enhances the antitumor activities of glycolipid-activated invariant NKT cells. J Immunol. 2009;182(5):2816-26. doi:10.4049/jimmunol.0803648.

4. Talay O, Shen $\mathrm{CH}$, Chen $\mathrm{L}$, Chen J. B7-H1 (PD-L1) on T cells is required for T-cell-mediated conditioning of dendritic cell maturation. Proc Natl Acad Sci USA. 2009;106(8):2741-6. doi:10.1073/pnas.0813367106.

5. Wang L, Pino-Lagos K, de Vries VC, Guleria I, Sayegh MH, Noelle RJ. Programmed death 1 ligand signaling regulates the generation of adaptive Foxp3+CD4+regulatory T cells. Proc Natl Acad Sci USA. 2008;105(27):9331-6. doi:10.1073/pnas.0710441105.

6. Postow MA, Callahan MK, Wolchok JD. Immune checkpoint blockade in cancer therapy. J Clinl Oncol Off J Am Soc Clin Oncol. 2015;. doi:10.1200/ JCO.2014.59.4358.

7. Merelli B, Massi D, Cattaneo L, Mandala M. Targeting the PD1/PD-L1 axis in melanoma: biological rationale, clinical challenges and opportunities. Crit Rev Oncol/Hematol. 2014;89(1):140-65. doi:10.1016/j. critrevonc.2013.08.002.

8. Thompson RH, Gillett MD, Cheville JC, Lohse CM, Dong H, Webster WS, et al. Costimulatory B7-H1 in renal cell carcinoma patients: indicator of tumor aggressiveness and potential therapeutic target. Proc Natl Acad Sci USA. 2004;101(49):17174-9. doi:10.1073/pnas.0406351101. 
9. Hamanishi J, Mandai M, Iwasaki M, Okazaki T, Tanaka Y, Yamaguchi K, et al. Programmed cell death 1 ligand 1 and tumor-infiltrating CD8+ $T$ lymphocytes are prognostic factors of human ovarian cancer. Proc Natl Acad Sci USA. 2007;104(9):3360-5. doi:10.1073/pnas.0611533104.

10. Inman BA, Sebo TJ, Frigola X, Dong H, Bergstralh EJ, Frank I, et al. PD-L1 (B7-H1) expression by urothelial carcinoma of the bladder and BCGinduced granulomata: associations with localized stage progression. Cancer. 2007;109(8):1499-505. doi:10.1002/cncr.22588.

11. Nakanishi J, Wada Y, Matsumoto K, Azuma M, Kikuchi K, Ueda S. Overexpression of B7-H1 (PD-L1) significantly associates with tumor grade and postoperative prognosis in human urothelial cancers. Cancer Immunol Immunother CII. 2007;56(8):1173-82. doi:10.1007/s00262-006-0266-z.

12. Nomi T, Sho M, Akahori T, Hamada K, Kubo A, Kanehiro H, et al. Clinical significance and therapeutic potential of the programmed death-1 ligand/programmed death-1 pathway in human pancreatic cancer. Clin Cancer Res Off J Am Assoc Cancer Res. 2007;13(7):2151-7. doi:10.1158/1078-0432.CCR-06-2746.

13. Zhao JM, Wu CP, Wang RC, Xu J, Zhu J, Wei J, et al. Expression and clinical significance of costimulatory molecule B7-H3 mRNA and B7-H3 protein in gastric carcinoma. Zhonghua wei chang wai ke za zhi = Chin J Gastrointest Surg. 2007;10(5):458-62.

14. Brown JA, Dorfman DM, Ma FR, Sullivan EL, Munoz O, Wood CR, et al, Blockade of programmed death-1 ligands on dendritic cells enhances $T$ cell activation and cytokine production. J Immunol. 2003;170(3):1257-66.

15. Dong H, Strome SE, Salomao DR, Tamura H, Hirano F, Flies DB, et al. Tumor-associated B7-H1 promotes T-cell apoptosis: a potential mechanism of immune evasion. Nat Med. 2002;8(8):793-800. doi:10.1038/ $\mathrm{nm} 730$.

16. Konishi J, Yamazaki K, Azuma M, Kinoshita I, Dosaka-Akita H, Nishimura M. B7-H1 expression on non-small cell lung cancer cells and its relationship with tumor-infiltrating lymphocytes and their PD-1 expression. Clin Cancer Res Off J Am Assoc Cancer Res. 2004;10(15):5094-100. doi:10.1158/1078-0432 CCR-04-0428.

17. Thompson RH, Gillett MD, Cheville JC, Lohse CM, Dong H, Webster WS, et al. Costimulatory molecule $\mathrm{B} 7-\mathrm{H} 1$ in primary and metastatic clear cell renal cell carcinoma. Cancer. 2005;104(10):2084-91. doi:10.1002/ cncr.21470.

18. Thompson RH, Kuntz SM, Leibovich BC, Dong H, Lohse CM, Webster WS, et al. Tumor B7-H1 is associated with poor prognosis in renal cell carcinoma patients with long-term follow-up. Cancer Res. 2006;66(7):3381-5. doi:10.1158/0008-5472.can-05-4303.

19. Hirano F, Kaneko K, Tamura H, Dong H, Wang S, Ichikawa M, et al. Blockade of B7-H1 and PD-1 by monoclonal antibodies potentiates cancer therapeutic immunity. Cancer Res. 2005;65(3):1089-96 (65/3/1089 [pii]).

20. Azuma T, Yao S, Zhu G, Flies AS, Flies SJ, Chen L. B7-H1 is a ubiquitous antiapoptotic receptor on cancer cells. Blood. 2008;111(7):3635-43. doi:10.1182/blood-2007-11-123141.

21. Ramsay AJ, Dong Y, Hunt ML, Linn M, Samaratunga H, Clements JA, et al. Kallikrein-related peptidase 4 (KLK4) initiates intracellular signaling via protease-activated receptors (PARs). KLK4 and PAR-2 are co-expressed during prostate cancer progression. J Biol Chem. 2008;283(18):12293304. doi:10.1074/jbc.M709493200.

22. Greenwald RJ, Freeman GJ, Sharpe AH. The B7 family revisited. Annu Rev Immunol. 2005;23:515-48. doi:10.1146/annurev. immunol.23.021704.115611.

23. Okazaki T, Honjo T. The PD-1-PD-L pathway in immunological tolerance. Trends Immunol. 2006;27(4):195-201. doi:10.1016/j.it.2006.02.001.

24. Fourcade J, Kudela P, Sun Z, Shen H, Land SR, Lenzner D, et al. PD-1 is a regulator of NY-ESO-1-specific CD8+ T cell expansion in melanoma patients. J Immunol (Baltim Md 1950). 2009;182(9):5240-9. doi:10.4049/ jimmunol.0803245.

25. Strome SE, Dong H, Tamura H, Voss SG, Flies DB, Tamada K, et al. B7-H1 blockade augments adoptive T-cell immunotherapy for squamous cell carcinoma. Cancer Res. 2003;63(19):6501-5.

26. Hino R, Kabashima K, Kato Y, Yagi H, Nakamura M, Honjo T, et al. Tumor cell expression of programmed cell death-1 ligand 1 is a prognostic factor for malignant melanoma. Cancer. 2010;116(7):1757-66. doi:10.1002/ cncr.24899.

27. Robert C, Ribas A, Wolchok JD, Hodi FS, Hamid O, Kefford R, et al. Anti-programmed-death-receptor-1 treatment with pembrolizumab in ipilimumab-refractory advanced melanoma: a randomised dosecomparison cohort of a phase 1 trial. Lancet. 2014;384(9948):1109-17. doi:10.1016/S0140-6736(14)60958-2.

28. Ribas A, Hodi FS, Kefford R, editors. Efficacy and safety of the anti-PD-1 monoclonal antibody MK-3475 in 411 patients (pts) with melanoma (MEL). In: 50th annual meeting of the American society of clinical oncology; 2014.

29. Kefford R, Ribas A, Hamid O, editors. Clinical efficacy and correlation with tumor PD-L1 expression in patients (pts) with melanoma (MEL) treated with the anti-PD-1 monoclonal antibody MK-3475. In: 50th annual meeting of the American society of clinical oncology, Chicago; 2014.

30. Raynaud FI, Orr RM, Goddard PM, Lacey HA, Lancashire H, Judson IR, et al. Pharmacokinetics of G3139, a phosphorothioate oligodeoxynucleotide antisense to bcl-2, after intravenous administration or continuous subcutaneous infusion to mice. J Pharmacol Exp Ther. 1997;281(1):420-7.

31. Weber JS MD, D'Angelo SP, et al. A phase 3 randomized, open-label study of nivolumab (anti-PD-1; BMS-936558; ONO-4538) versus investigator's choice chemotherapy (ICC) in patients with advanced melanoma after prior anti-CTLA-4 therapy. In: Program and abstracts from the 39th European society for medical oncology congress, Madrid; 2014. (Abstract

\section{LBA3_PR)}

32. Robert C, Long GV, Brady B, Dutriaux C, Maio M, Mortier L, et al. Nivolumab in previously untreated melanoma without BRAF mutation. N Engl J Med. 2015;372(4):320-30. doi:10.1056/NEJMoa1412082

33. Tumeh PC, Harview CL, Yearley JH, Shintaku IP, Taylor EJ, Robert L, et al. PD-1 blockade induces responses by inhibiting adaptive immune resistance. Nature. 2014;515(7528):568-71. doi:10.1038/nature13954.

34. Kefford RRA, Hamid O, et al. Clinical efficacy and correlation with tumor PD-L1 expression in patients with melanoma treated with the anti-PD-1 monoclonal antibody pembrolizumab (MK-3475). In: The 2014 ASCO annual meeting; 2014

35. Balch CM, Buzaid AC, Soong SJ, Atkins MB, Cascinelli N, Coit DG, et al. Final version of the American Joint Committee on Cancer staging system for cutaneous melanoma. J Clin Oncol. 2001;19(16):3635-48.

36. Balch CM, Soong SJ, Murad TM, Ingalls AL, Maddox WA. A multifactorial analysis of melanoma: III. Prognostic factors in melanoma patients with lymph node metastases (stage II). Ann Surg. 1981;193(3):377-88.

37. Balch CM, Soong SJ, Gershenwald JE, Thompson JF, Reintgen DS, Cascinelli $\mathrm{N}$, et al. Prognostic factors analysis of 17,600 melanoma patients: validation of the American Joint Committee on Cancer melanoma staging system. J Clin Oncol Off J Am Soc Clin Oncol. 2001;19(16):3622-34.

38. Balch CM, Gershenwald JE, Soong SJ, Thompson JF, Atkins MB, Byrd DR, et al. Final version of 2009 AJCC melanoma staging and classification. J Clin Oncol Off J Am Soc Clin Oncol. 2009;27(36):6199-206. doi:10.1200/ JCO.2009.23.4799.

39. Tarhini AA, Thalanayar PM. Melanoma adjuvant therapy. Hematol Oncol Clin N Am. 2014;28(3):471-89. doi:10.1016/j.hoc.2014.02.004.

40. Topalian SL, Hodi FS, Brahmer JR, Gettinger SN, Smith DC, McDermott DF, et al. Safety, activity, and immune correlates of anti-PD-1 antibody in cancer. N Engl J Med. 2012;366(26):2443-54. doi:10.1056/NEJMoa1200690.

41. Weber JS, Kudchadkar RR, Yu B, Gallenstein D, Horak CE, Inzunza HD, et al. Safety, efficacy, and biomarkers of nivolumab with vaccine in ipilimumab-refractory or -naive melanoma. J Clin Oncol Off J Am Soc Clin Oncol. 2013;31(34):4311-8. doi:10.1200/JCO.2013.51.4802.

42. Garon EB, Rizvi NA, Hui R, Leighl N, Balmanoukian AS, Eder JP, et al. Pembrolizumab for the treatment of non-small-cell lung cancer. N Engl J Med. 2015;372(21):2018-28. doi:10.1056/NEJMoa1501824.

43. Hodi FSSM, Kluger HM, et al. Long-term survival of ipilimumab-naïve patients (pts) with advanced melanoma (MEL) treated with nivolumab (anti-PD-1; BMS-936558; ONO-4538) in a phase I trial. In: The 2014 ASCO annual meeting; 2014

44. Gettinger SSF, Antonia SJ, et al. First-line nivolumab (anti-PD-1; BMS936558, ONO-4538) monotherapy in advanced non-small cell lung cancer (NSCLC): safety, efficacy, and correlation of outcomes with PD-L1 status. In: The 2014 ASCO annual meeting; 2014.

45. Roda JM, Parihar R, Magro C, Nuovo GJ, Tridandapani S, Carson WE 3rd. Natural killer cells produce T cell-recruiting chemokines in response to antibody-coated tumor cells. Cancer Res. 2006;66(1):517-26. doi:10.1158/0008-5472.CAN-05-2429. 